Background Men who have sex with men (MSM) have one of the highest HIV prevalence rates of any high risk population in Central America. Since 2007, the Sentinel Surveillance and Control of Sexually Transmitted Infections (VICITS) strategy has been implemented in two public health clinics targeting MSM in Guatemala City. This combination prevention strategy provides diagnosis and treatment of STIs, HIV testing, condom distribution, and educational activities.

Method From 2007 to 2012, MSM were recruited through the Internet or by community-based organisation staff to attend two VICITS clinics in Guatemala City. Clinic attendees were asked demographic and behavioural information and tested for HIV and syphilis. Variables included in trend analysis included HIV and syphilis test results and condom use with casual partner in the past month. All analyses were performed using Stata 9.0.

Results A total of 433 MSM attended VICITS clinics from 2007 to 2012 . HIV prevalence was $10.3 \%$ from $2009-2010$ ( $n=117$, 95\% CI: $5.4-17.2)$ and $11.7 \%$ from $2011-2012(\mathrm{n}=179,95 \% \mathrm{CI}$ : 7.4-17.4). Syphilis prevalence was $6.6 \%$ from $2009-2010$ ( $n=106$, 95\% CI: 2.7-13.1) and 5.9\% from 2011-2012 ( $\mathrm{n}=153$, 95\% CI: 2.7-10.9). Consistent condom use with a casual partner in the last month was $61.9 \%(\mathrm{n}=56,95 \%$ CI: 49.1-74.7) from 20092010 and $62.7 \%$ ( $n=72$, 95\% CI: 51.7-73.8) from 2011-2012. No significant changes in attendance from 2007 to 2012 were noted at both clinics.

Discussion No changes in HIV and syphilis prevalence among MSM attending VICITS clinics in Guatemala City were observed. Consistent condom use should be continued to be emphasised in casual relationships. Routine monitoring of sentinel surveillance data allows for timely feedback of HIV prevention services and provides key information for early HIV diagnosis, as an intervention that has shown evidence of effectiveness programme.

\section{P3.433 COST-EFFECTIVENESS OF ENHANCED SYPHILIS SCREENING AMONG HIV-POSITIVE MEN WHO HAVE SEX WITH MEN}

doi:10.1136/sextrans-2013-051184.0884

${ }^{1}$ A Tuite, ${ }^{1} \mathrm{M}$ Machina, ${ }^{2} \mathrm{~A}$ Burchell, ${ }^{1} \mathrm{~A}$ Bayoumi, ${ }^{1} \mathrm{D}$ Fisman. ${ }^{1}$ University of Toronto, Toronto, ON, Canada; ${ }^{2}$ Ontario HIV Treatment Network, Toronto, ON, Canada

Background Syphilis co-infection has increased substantially among HIV-positive men who have sex with men (MSM). Screening rates in this population remain below recommended guidelines for screening frequency. We evaluated the cost-effectiveness of increasing the frequency and coverage of screening to recommended levels in HIV-positive MSM receiving HIV care.

Methods We developed a Markov cohort model to evaluate the cost-effectiveness of enhanced syphilis screening in a cohort of HIVpositive MSM with baseline characteristics (including current screening coverage) reflective of men enrolled in an observational cohort study of individuals receiving medical care for HIV in Ontario, Canada. We compared usual care $50 \%$ of the population screened annually) to enhanced screening (100\% screened every 3 months). Model parameters were from the health literature and the observational cohort database. Both strategies incorporated a treponemal screening test followed by a confirmatory non-treponemal test; test characteristics varied at each stage of syphilis infection. Outcomes were quality-adjusted life years (OALYs), lifetime costs (2011 Canadian dollars), and incremental cost-effectiveness ratios. The model used the perspective of a public health care payer and a lifetime time horizon, with a base case discount rate of $5 \%$ applied to future costs and outcomes.

Results Compared to usual care, enhanced screening increased quality-adjusted life expectancy by 0.016 OALYs and decreased costs by $\$ 1437$ per person. Enhanced screening remained the preferred strategy when the incidence of syphilis was above 0.5 per 100 person-years. Findings were robust in wide-ranging deterministic sensitivity analyses, including assumptions around reduced adherence to screening recommendations. In probabilistic sensitivity analysis, the probability that enhanced screening was cost-effective exceeded $85 \%$, assuming a willingness-to-pay of up to $\$ 50,000$ per OALY.

Conclusions Our model suggests that in populations with moderate to high rates of syphilis acquisition, enhanced syphilis screening alongside HIV medical care has the potential to improve health and save costs.

\section{P3.434 WITHDRAWN BY AUTHOR}

\section{P3.435 OPT-OUT RECTAL SCREENING FOR CHLAMYDIA AND GONORRHEA IN YOUNG MEN WHO HAVE SEX WITH MEN (YMSM)}

doi:10.1136/sextrans-2013-051184.0885

G Hart-Cooper, K Owusu-Edusei, H Chesson, K Hoover. Centers for Disease Control and Prevention, Atlanta, GA, United States

Objective In the United States, almost $20 \%$ of new HIV infections in 2010 occurred in young MSM (YMSM) aged 13-24 years old. YMSM also have high rates of other sexually transmitted infections (STIs). STIs can facilitate HIV transmission and acquisition, and indicate unsafe sexual behaviour that might be conducive to HIV transmission. CDC recommends risk-based rectal chlamydia and gonorrhoea screening of MSM who report receptive anal intercourse (RAI), to protect their health and prevent HIV transmission. However, screening rates have been low. Opt-out screening of all YMSM during clinical encounters might be an effective approach to STI screening.

Methods We used a cohort decision analysis model to compare two screening algorithms of YMSM aged 18-24 years: (1) risk-based screening of YMSM who report RAI, and (2) screening YMSM unless they opt-out. Based on published data, we assumed that $61 \%$ of YMSM disclosed same-sex behaviour to providers and that $67 \%$ of YMSM had RAI. Among YMSM who had RAI, 16\% underwent risk-based rectal screening for chlamydia and/or gonorrhoea, and the prevalence of infection was $12 \%$. Outcomes included the number of rectal infections identified and number of tests required to identify one infection.

Results Among 1000 YMSM, opt-out screening identified 59\% of infections versus $16 \%$ with risk-based screening. Opt-out screening required 10.4 tests to identify one infection versus 8.4 with riskbased screening. Both screening approaches missed 39\% of infections because providers were unaware that patients were MSM.

Conclusions Although opt-out screening required two additional tests to identify one case, it identified 3.7 times more infections. Increased diagnosis and treatment of STIs can lead to decreased STI prevalence and HIV transmission, facilitate provider interventions for at-risk youth, and increase patient awareness of the risks associated with unsafe sexual practises. Increased awareness of patients sexual behaviour among providers is needed to provide optimal care.

\section{P3.436 IMPROVING STRATEGIES IN IDENTIFYING TRANSGENDER WOMEN CLIENTS TOWARDS DATA DISAGGREGATION IN THE PHILIPPINE INTEGRATED HIV BEHAVIOURAL AND SEROLOGICAL SURVEILLANCE (IHBSS)}

doi:10.1136/sextrans-2013-051184.0886

R N Cortes. Philippine NGO Council on Population, Health and Welfare, Inc., Pasay City, Philippines

Since 2007, the Philippine Integrated HIV Behavioral and Serological Surveillance (IHBSS) still lump the transgender (TG) women population with men having sex with men (MSM), which is a 
socio-political issue among empowered Filipino TG women. Recognizing similar sexual behaviour risks among MSM and TG women, the latter associated with body modifications concept(i.e. hormone replacement, collagen injections/implants) have a heightened risk due to unsafe and improper injecting practises. Thus, the ISEANHivos Program (IHP) (a Global Fund Multi-country Grant) in the Philippines, through consultations with the TG community, aims to develop an operational TG definition that would hopefully be feasible in a national surveillance.

The method used was the conduct of four (4) focus group discussions (FGDs) with TG women members (with 5-10 participants each FGD) from community-based organisations (CBOs) in Metro Manila, Cebu City and Davao City.

The findings revealed that majority of the participants do not generally agree with the three (3) proposed qualifiers/criteria in identifying TG women used in the IHBSS 2011 MSM questionnaire. "Looks like a female" is subjective; "Taking/injecting hormones" is not necessary; and those who have "undergone sex reassignment surgery" would identify themselves as a non-TG woman. Some of the suggestions are the inclusion of a time element in one's gender identification/expression to eliminate those who just occasionally identifies/express themselves as female; consider any form of affirming one's identity as a woman; and the use of three (3) questions - asking one's sex assigned birth, gender identity and gender expression.

The study concludes with IHP-Philippines' proposed operational definition of TG women: those whose sex assigned birth is male; and whose gender identity is generally female and/or whose gender expression is generally feminine. They need not undergo any form of body modification, may have varying sexual orientations, and may self-identify using various local gender terms.

\section{P3.437 HARVAT: SEX WORK INVOLVEMENT AND ASSOCIATED OCCUPATIONAL RISKS AMONG SELECTED TRANSGENDER WOMEN IN CEBU CITY, PHILIPPINES}

doi:10.1136/sextrans-2013-051184.0887

R N Cortes. Philippine NGO Council on Population, Health and Welfare, Inc., Pasay City, Philippines

The expansion of sex work (or harvat in Cebu) in the Philippines has surfaced transgender (TG) escorts and online-based sex workers (SWs), who are generally exposed to health risks such as sexually transmitted infections (STIs), HIV/AIDS. Recent Philippine Integrated HIV Behavioral and Serological Surveillance (IHBSS) 2011 showed Cebu City having the second highest HIV prevalence among men who have sex with men (MSM) (i.e. 4.7 percent); which TG women are included. Due to the lack of social recognition of TG women, combined with the stigmatisation of SWs, this leads to discrimination, violence and health risks among TG women SWs. Hence, it is important to look at their gender identity and selfdescription; context and nature of sex work involvement; and their perceived/experienced occupational risks.

The method used was face-to-face in-depth interviews with fifteen (15) TG women SWs in Cebu City as identified by the researcher. Content analysis was used for qualitative analysis of transcribed interviews. The findings revealed that TG women SWs do not necessarily identify themselves as TG but see themselves as women expressed in different modalities, but commonly undergoing body modifications (i.e. hormone pills, collagen injections). Economic benefits and sexual enjoyment gained are the main motivations for TG women SWs, which is related to greater preference for foreigner clients and "versatile" sexual role. Escorting has the most economic benefits, followed by online chatting and online harvat. Lastly, there were lesser experiences of occupational risks (i.e. health, abuse, legal) than what they perceived.
The study concludes that perceived/experienced risks associated with sex work are not merely occupational, but are also behavioural and gender-based. As such, sexual behaviour and not their occupation as SWs make TG women at risk to STIs, HIV/AIDS. Hence, HIV education and prevention strategies should be client-specific to address health needs of the general TG women population.

\section{P3.438 CHALLENGES IN MANAGING TRANSGENDER INDIVIDUALS - OBSERVATIONS IN A UK SEXUAL HEALTH CLINIC}

doi:10.1136/sextrans-2013-051184.0888

H Roberts, K A Fernando, H Jaleel. Southend University Hospital NHS Foundation Trust, Essex, UK

Background Transgender individuals may have complex medical and psychosocial needs. Prior studies have reported an increased incidence of STIs including HIV. Low self esteem, social isolation, self-harm and suicide have been observed with greater frequency. Additionally, a greater likelihood of being subject to relationship violence and sexual assault is recognised. Discrimination in school or the work-place, and even in healthcare settings adds to psychological burden. Healthcare seeking behaviour of transgender individuals may, therefore, be adversely affected.

Cases: We share experiences of two transgender patients and their health concerns.

The first underwent gender reassignment 20 years ago (aged 19) She reported psychological problems including depression, anxiety attacks and a prior suicide attempt. In 2010 she was diagnosed with HIV when presented with pneumocystis jirovecii pneumonia. Antiretroviral therapy (ART) management has been challenging with regards to exacerbation of psychological ill-health thought due to the ART agent, efavirenz. This was, therefore, substituted with rilpivirine, with favourable outcome. Reluctance to disclose her HIV status to her partner persists. Additionally, self-purchased 'hormonal' medication remains concerning with regards to unsupervised use and potential ART interactions.

The second patient underwent gender reassignment 15 years ago. She is HIV negative and presents with unusually frequent recurrences of anogenital warts, bacterial abscesses and herpetic ulcers. She also has a psychosexual disorder thought resulting from prior sexual abuse

Discussion Concern remains regarding lack of awareness amongst health care professionals (HCPs) with regards to the above complexities and the need for appropriate, empathic and non-judgemental management. Surveys of medical school curricula highlight that transgender health needs are often omitted, with limited professional information resources.

Conclusion HCPs must remain aware of the increased risk of mental and sexual ill-health. This will allow timely recognition of such problems and prompt intervention. Intervention may require collaborative interagency approaches with HCPs from mental health services.

\section{P3.439 UNDERSTANDING THE LOCALISED PERSPECTIVE ON TRANSGENDER WOMEN FOR DEVELOPING TRANSGENDER- SPECIFIC HEALTH SERVICES AND HIV PREVENTION PROGRAMMES IN THE PHILIPPINES}

doi:10.1136/sextrans-2013-051184.0889

R N Cortes. Philippine NGO Council on Population, Health and Welfare, Inc., Pasay City, Philippines

The Philippines do not have localised term to refer to transgender (TG) persons. In fact, the common local terms "bakla", "bading" and "bayot" are negatively used to refer to TG women. Even the Philippine Integrated HIV Behavioral and Serological Surveillance (IHBSS) do not disaggregate data for men having sex with men (MSM) and 